\title{
Prevalence of Fever in Patients Undergoing Left Atrial Ablation of Atrial Fibrillation Guided by Barium Esophagraphy
}

\author{
RUBINDER S. RUBY, M.D., DARRYL WELLS, M.D., SUNDAR SANKARAN, M.D., \\ ERIC GOOD, D.O., KRIT JONGNARANGSIN, M.D., MATTHEW EBINGER, D.O., \\ FRANK BOGUN, M.D., FRANK PELOSI JR., M.D., HAKAN ORAL, M.D., FRED MORADY, M.D., \\ and AMAN CHUGH, M.D.
}

From the Section of Electrophysiology/Division of Cardiology, University of Michigan Hospitals, Ann Arbor, Michigan, USA

Fever in Patients Undergoing LA Ablation of AF Guided by Barium Esophagraphy. Background: Real-time esophageal imaging is critical in avoiding esophageal injury. However, the safety of esophageal imaging with barium has not been specifically explored.

Methods: Three hundred seventy consecutive patients underwent left atrial (LA) ablation of atrial fibrillation (AF) under conscious sedation. One hundred eighty-five patients $(50 \%)$ underwent the ablation procedure with, and 185 patients $(50 \%)$ underwent the procedure without administration of barium. Fever, as a surrogate for aspiration, was defined as a maximal temperature $\geq 100^{\circ} \mathrm{F}$ within the first 24 hours following the ablation procedure.

Results: Thirty of the 370 patients $(8 \%)$ developed fever within 24 hours after LA ablation. The prevalence of fever was $9 \%(17 / 185)$ among patients who received barium and $7 \%(13 / 185)$ among those who did not receive barium $(P=0.6)$. Evaluation revealed the following causes of fever in 14 of the 30 patients $(47 \%)$ with no difference in prevalence between the 2 groups: pericarditis, venous thromboembolism, hematoma, and infiltrate on chest radiography. Multivariate analysis failed to reveal any factors associated with development of fever. None of the patients experienced serious complications such as respiratory failure or atrioesophageal fistula.

Conclusions: Fever may occur in approximately $10 \%$ of patients undergoing LA ablation of AF. Administration of barium is not associated with fever or other complications such as aspiration pneumonia. Real-time imaging of the esophagus with barium administration in conjunction with conscious sedation appears to be safe. (J Cardiovasc Electrophysiol, Vol. 20, pp. 883-887, August 2009)

catheter ablation, atrial fibrillation, esophagus, barium, aspiration

\section{Introduction}

Real-time esophageal imaging is critical in avoiding esophageal injury during left atrial (LA) ablation of atrial fibrillation (AF). ${ }^{1,2}$ Various strategies have been employed to minimize the risk of esophageal injury and formation of an atrioesophageal (AE) fistula, including the administration of oral barium during the ablation procedure. ${ }^{3}$ Barium esophagraphy delineates the course of the esophagus, which is then avoided during ablation of the posterior LA.

A possible concern with barium administration is aspiration pneumonitis in patients undergoing the procedure under conscious sedation. Pulmonary aspiration may be charac-

Dr. R.S. Ruby and Dr. D. Wells contributed equally to this manuscript.

Dr. Pelosi reports receiving research grants and compensation for participation on a speaker's bureau from Boston Scientific and Medtronic. Dr. Oral reports ownership or partnership interest, stock options and/or other rights in a patent or intellectual property for Ablation Frontiers, Inc. The other authors report no conflicts.

Address for correspondence: Aman Chugh, M.D., Cardiovascular Center, SPC 5853, 1500 East Medical Center Drive, Ann Arbor, MI 48109-5853, USA. Fax: 734-936-7026; E-mail: achugh@umich.edu

Manuscript received 1 September 2008; Revised manuscript received 28 January 2009; Accepted for publication 9 February 2009.

doi: $10.1111 / \mathrm{j} .1540-8167.2009 .01464 . \mathrm{x}$ terized by findings such as cough, pneumonitis, respiratory failure, fever, and rarely death. ${ }^{4}$ In this study, we evaluated the prevalence of fever within the first 24 hours as a surrogate for aspiration pneumonitis in patients who underwent catheter ablation of AF with and without barium administration.

\section{Methods}

The study protocol was approved by the Institutional Review Board. Three hundred seventy patients who underwent LA ablation of AF guided by a 3-dimensional (3D) mapping system (CARTO, Biosense-Webster, Diamond Bar, CA, USA) were evaluated retrospectively. In $185(50 \%)$ of these patients (control group), the procedure was performed prior to the use of a barium swallow. These patients underwent circumferential pulmonary vein ablation between June 2003 and January 2004 , as previously described. ${ }^{5}$ In another 185 patients (barium group), the procedure was guided by a barium swallow for real-time imaging of the esophagus. In the barium group, the ablation procedure involved circumferential pulmonary vein ablation in 15 patients $(8 \%)$, electrogramguided ablation in 46 patients $(25 \%),{ }^{6}$ pulmonary vein isolation in 7 patients $(4 \%)$, and various combinations of these strategies in the remaining 117 (63\%) of the patients. These patients underwent the ablation procedure between January and June, 2005. Barium swallow was performed with $5 \mathrm{cc}$ of barium paste ${ }^{3}$ (E-Z-Paste, E-Z-M Canada Inc., 
A

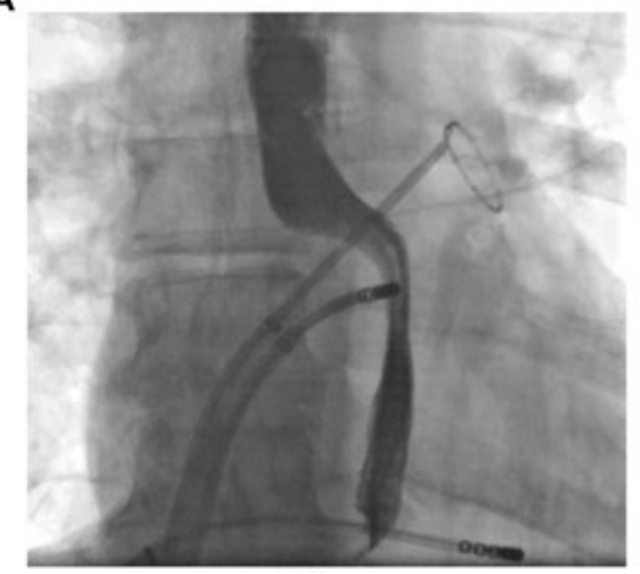

AP
B

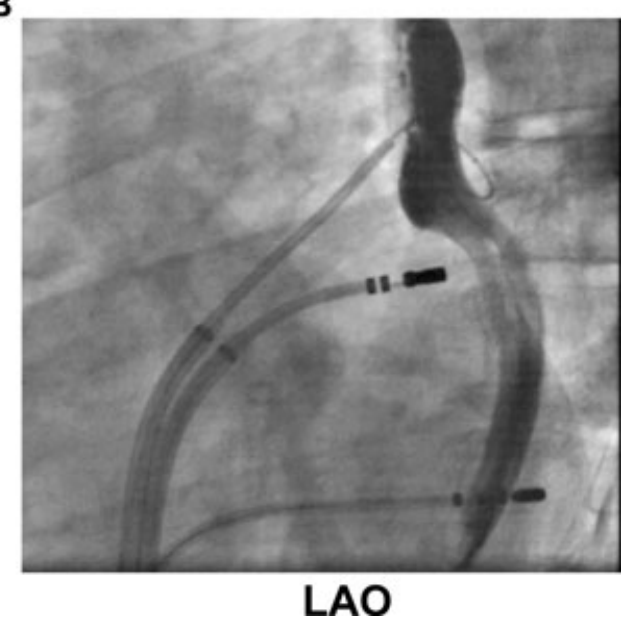

Figure 1. An esophagram obtained during a barium swallow in a patient undergoing an ablation procedure for atrial fibrillation (AF). The white arrows demarcate the course of the esophagus, which was then tagged on the 3D map. Also shown are the ablation catheter and the ring catheter in the left superior pulmonary vein (PV). (A) Anteroposterior view (AP). (B) Left anterior oblique view $\left(45^{\circ}\right)$.

Quebec, Canada) administered after creation of a 3D replica of the LA but prior to administration of medications for conscious sedation. Cine-fluoroscopic images of the esophagus during barium swallow were recorded in 2 views (Fig. 1), and the course of the esophagus was tagged on the $3 \mathrm{D}$ map. ${ }^{7}$ None of the patients underwent placement of a urinary catheter.

All patients were monitored overnight. Vital signs, including temperature, blood pressure, heart rate, and oxygen saturation were recorded on a flow sheet every 6 hours, for 24 hours. The flow sheet was reviewed for each of the 370 patients by 2 of the investigators (DW, RR). Since aspiration pneumonitis typically manifests itself within 2 hours of the procedure, ${ }^{4}$ fever was defined as an oral temperature $\geq 100^{\circ} \mathrm{F}$ within the first 24 hours following the ablation procedure. Any laboratory or radiologic evaluation for fever was also analyzed. The medical record was also reviewed for any complications related to the ablation procedure, such as those related to venous access, pericarditis, perforation, thromboembolism, and esophageal injury. Length of hospital stay (LOS) was also evaluated.

\section{Statistical Analysis}

Continuous data are expressed as mean \pm standard deviation and were compared with the Student's $t$-test. Categor-

\section{TABLE 1}

Baseline Clinical Characteristics of Total Study Population

\begin{tabular}{lccc}
\hline Variables & Barium $(\mathbf{n}=\mathbf{1 8 5})$ & Control $(\mathbf{n}=\mathbf{1 8 5})$ & P-Value \\
\hline Sex $(\mathrm{M} / \mathrm{F})$ & $137 / 48$ & $147 / 38$ & 0.2 \\
Age & $56 \pm 10$ & $154 \pm 11$ & 0.1 \\
Weight $(\mathrm{kg})$ & $94 \pm 21$ & $100 \pm 19$ & 0.1 \\
HTN & 74 & 75 & 0.9 \\
CAD & 16 & 7 & 0.08 \\
LVEF, $\%$ & $56 \pm 8$ & $55 \pm 9$ & 0.6 \\
LA, mm & $42 \pm 7$ & $42 \pm 6$ & 0.6 \\
Procedure time (min) & $215 \pm 44$ & $217 \pm 53$ & 0.8 \\
\hline
\end{tabular}

$\mathrm{CAD}=$ coronary artery disease; $\mathrm{F}=$ female; $\mathrm{HTN}=$ hypertension; $\mathrm{LA}=$ left atrial diameter; $\mathrm{LVEF}=$ left ventricular ejection fraction; $\mathrm{M}=$ male. ical data are expressed as proportions and compared with a 2 -sided Fisher's exact test. Logistic regression analysis was performed with age, gender, left ventricular ejection fraction (LVEF), LA diameter, use of barium, procedure time, and pericarditis as independent variables and fever as a dependent variable using SPSS 16.0 (Chicago, IL, USA). A P value $<0.05$ indicated statistical significance.

\section{Results}

There were no significant differences in baseline characteristics between the 2 groups (Table 1). Specifically, the procedure time for patients who underwent the ablation procedure with and without barium was $215 \pm 44$ minutes and $217 \pm 53$ minutes, respectively $(\mathrm{P}=0.8)$. Thirty of the 370 patients $(8 \%)$ developed fever following LA ablation. The prevalence of fever was 9\% (17/185) among patients who received barium, and 7\% (13/185) among those who did not receive barium $(P=0.57)$. Among clinical variables such as age, gender, LVEF, LA size, pericarditis, procedure time, and barium use, none was associated with the development of fever. There were no significant differences in baseline characteristics of patients who did and did not develop fever (Table 2). In one of the patients in the barium group (0.5\%), aspiration was documented on cinefluoroscopy during the ablation procedure (Fig. 2).

Table 3 shows the results of the evaluation for fever. A possible cause of fever was ascertained in 14 of the

\section{TABLE 2}

Baseline Clinical Characteristics of Patients with Fever

\begin{tabular}{lccc}
\hline Variables & $\begin{array}{c}\text { Barium } \\
(\mathbf{n = 1 7})\end{array}$ & $\begin{array}{c}\text { Control } \\
(\mathbf{n = 1 3})\end{array}$ & P-Value \\
\hline Sex $(\mathrm{M} / \mathrm{F})$ & $12 / 5$ & $11 / 2$ & 0.4 \\
Age & $56 \pm 9$ & $47 \pm 16$ & 0.1 \\
LVEF, \% & $59 \pm 6$ & $55 \pm 9$ & 0.2 \\
LA, mm & $44 \pm 4$ & $43 \pm 4$ & 0.4 \\
\hline
\end{tabular}

$\mathrm{F}=$ female; $\mathrm{LA}=$ left atrial diameter; $\mathrm{LVEF}=$ left ventricular ejection fraction; $M=$ male. 


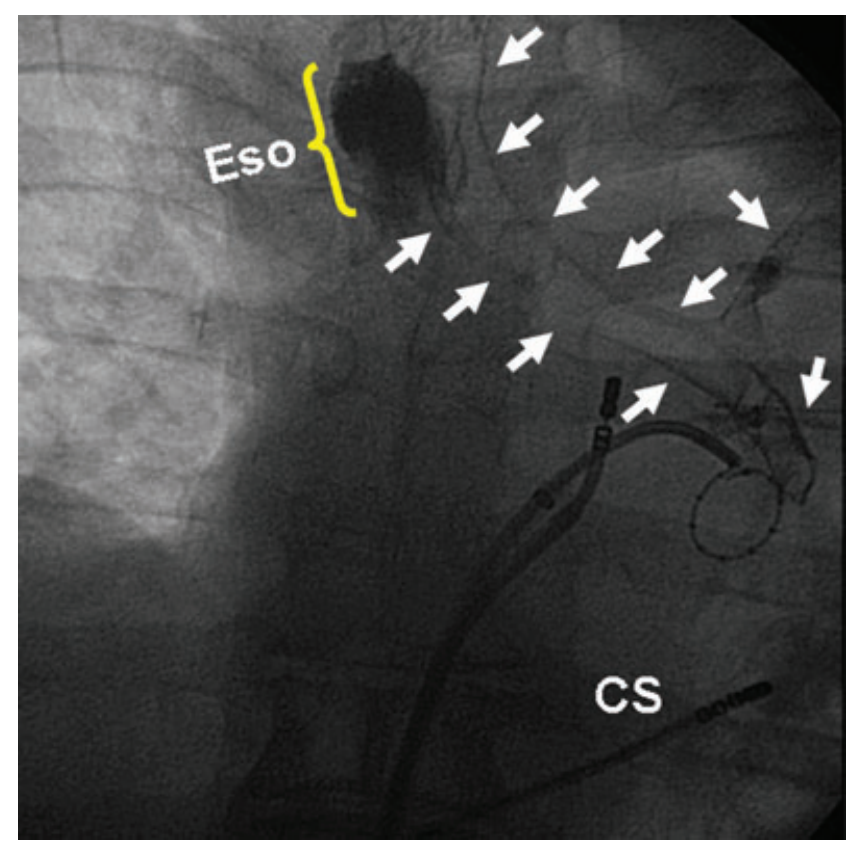

Figure 2. Aspiration of barium. This inadvertent bronchogram (arrows, AP view) was noted incidentally during the ablation procedure. The patient remained asymptomatic during and after the ablation procedure, and did not develop pyrexia. A chest radiograph on the morning after the ablation procedure was normal. The yellow bracket highlights the barium paste within the esophagus. Also shown are the ablation catheter during energy delivery at the roof, and the ring catheter in the left inferior PV.

30 patients: pericarditis in 7 patients, access site hematoma in 3 patients, pulmonary infiltrate in 2 patients (Fig. 3), and venous thromboembolism in 2 patients. There was leukocytosis in 7 patients $(23 \%)$. Blood cultures were obtained in 7 patients (23\%) and were negative in all. Urinalysis was normal in each of the 10 patients in whom it was obtained.

Empiric antibiotics were prescribed in 3 patients (10\%). The mean LOS was $2 \pm 1.5$ and $1.9 \pm 1.4$ days among patients who developed fever in the barium and control groups, respectively $(\mathrm{P}=0.9)$. No patient developed respiratory failure or AE fistula or required prolonged hospitalization. There

TABLE 3

Comparison of Complications in Patients with Fever

\begin{tabular}{lccc}
\hline Variables & $\begin{array}{c}\text { Barium } \\
(\mathbf{n}=\mathbf{1 7})\end{array}$ & $\begin{array}{c}\text { Control } \\
(\mathbf{n = 1 3})\end{array}$ & P-Value \\
\hline Temperature, mean $\pm(\mathrm{SD})$ & $100.6 \pm 0.4^{\circ} \mathrm{F}$ & $100.8 \pm 0.7^{\circ} \mathrm{F}$ & 0.3 \\
Pericarditis, $\mathrm{n}(\%)$ & $4(24)$ & $3(23)$ & 1 \\
Infiltrate, n (\%) & $1(6)$ & $1(8)$ & 1 \\
Pulmonary embolism, n (\%) & $0(0)$ & $1(8)$ & 0.3 \\
Hematoma, n (\%) & $2(12)$ & $1(8)$ & 1 \\
DVT, n (\%) & $1(6)$ & $1(8)$ & 1 \\
Heart failure, n (\%) & $2(12)$ & $0(0)$ & 0.5 \\
Neurological events, n (\%) & $1(6)$ & $0(0)$ & 1 \\
Blood cultures done, n (\%) & $4(24)$ & $3(23)$ & 1 \\
Blood cultures positive, $\mathrm{n}(\%)$ & 0 & 0 & $\mathrm{NA}$ \\
Antibiotics on discharge, $\mathrm{n}(\%)$ & $1(6)$ & $2(15)$ & 0.6 \\
Oxygen requirement, n (\%) & $3(18)$ & $2(15)$ & 1 \\
Readmit, n (\%) & $1(6)$ & 0 & 1 \\
LOS (days) & $2 \pm 1.5$ & $1.9 \pm 1.4$ & 0.9 \\
\hline CT & & &
\end{tabular}

$\mathrm{CT}=$ computed tomography; DVT $=$ deep vein thrombosis; $\mathrm{LOS}=$ length of stay; $\mathrm{NA}=$ not applicable.

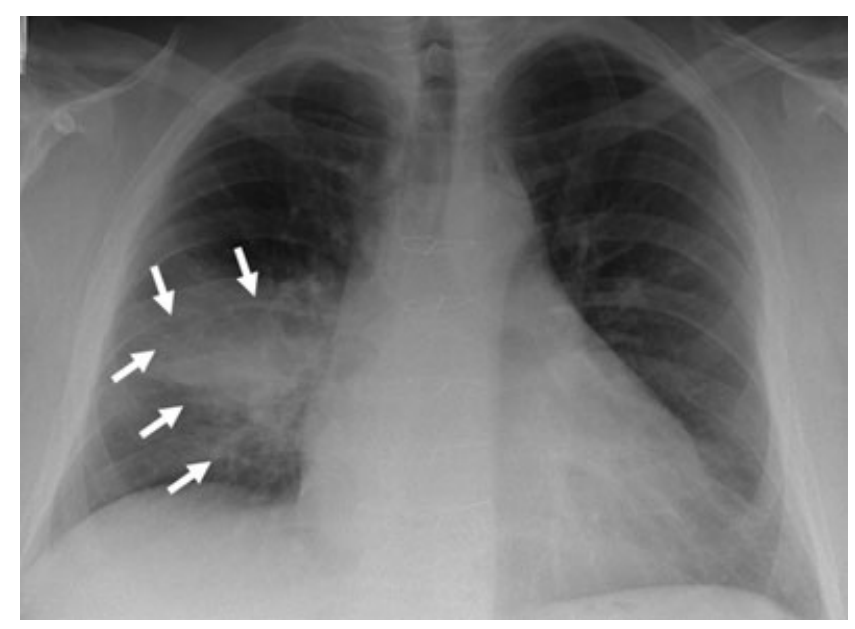

Figure 3. A chest radiograph (PA) from a patient who did not receive barium and subsequently developed fever on the morning after the ablation procedure. The arrow points to an infiltrate in the right lower lobe, which was likely due to aspiration. The patient was prescribed oral antibiotics and he recovered uneventfully. Follow-up imaging showed resolution of the infiltrate.

were no differences in the rate of complications between the 2 groups. One of the patients in the barium group presented to an emergency room at another hospital for complications related to an access-site hematoma. Another patient in the barium group was readmitted 24 hours after discharge for pericarditis that responded to nonsteroidal medications. None of the patients with fever within 24 hours of the ablation procedure developed recurrent fever or pulmonary complications following discharge.

\section{Discussion}

\section{Main Findings}

The main finding of this study is that there is no difference in the prevalence of postablation fever in patients undergoing $\mathrm{LA}$ ablation of AF with or without the guidance of a barium esophagram. A barium swallow allows for real-time imaging of the esophagus, which then aids the operator in the avoidance of the esophagus during the ablation procedure. A concern for respiratory failure due to barium aspiration has been used as an argument against using this technique. This study demonstrates that barium administration does not increase the risk of fever or other complications as compared to a control group. The results of the study should be reassuring to electrophysiologists and patients that real-time esophageal imaging does not appear to add to the risk of the ablation procedure.

\section{Risk Factors for Aspiration}

The results of this study do not imply that aspiration of barium does not occur in patients undergoing the procedure guided by an esophagram. Indeed, aspiration of barium has been documented in a small number of patients in our experience, as shown in Figure 2. Of course, aspiration is not unique to patients receiving barium and was documented in a patient who did not receive barium (Fig. 3). Patients may aspirate secretions or gastric contents during any procedure requiring sedation. ${ }^{4}$ A lack of any serious sequalae in the 
study patients implies that the amount of aspirate is small and may be "subclinical." It is likely that barium administration simply facilitates the visualization of the aspirate on fluoroscopy.

The risk factors for aspiration pneumonitis during a catheter ablation procedure guided by an esophagram include the level of sedation, patient's anatomic profile, comorbid conditions, ${ }^{4}$ and amount of barium. It is likely that the very small amount of administered barium, and even a lesser amount that may be rarely aspirated, minimizes the risk of serious consequences. If aspiration has been documented, irrespective of barium administration, it may be reasonable to treat the patient with a short course of antibiotics.

\section{Causes of Fever}

The differential diagnosis in patients with fever following an ablation procedure is legion. The possibilities include atelectasis, pneumonitis, pericarditis, hematoma at the access site, venous thromboembolism, thrombophlebitis, urinary tract infection (in patients who undergo urinary catheterization) and rarely, esophageal injury. Indeed, many of these conditions were observed in study patients who developed fever. This study may thus provide a framework in the evaluation and management of such patients.

The most dire implication in a patient presenting with fever following an ablation procedure for $\mathrm{AF}$ is that of an $\mathrm{AE}$ fistula. This possibility mandates an emergent work up and appropriate consultation in order to prevent severe complications and death. The evaluation should include an urgent computed tomographic examination to evaluate the left atrium and esophagus. The electrophysiologist should work closely with an experienced radiologist and obtain emergent surgical consultation if the scan is abnormal. However, if radiofrequency energy was not delivered over the esophagus as visualized on the esophagram, the clinician may be reassured, and other possibilities may be pursued. It should be noted that patients with an AE fistula may not present with classic symptoms of fever and odynophagia, and may present with nonspecific findings as well. ${ }^{8}$

\section{Alternatives to Esophagraphy}

A variety of other strategies have been used in an attempt to reduce the risk of serious injury to the esophagus during the ablation procedure for AF. These include insertion of a radioopaque naso- or oro-gastric tube, ${ }^{9}$ esophageal temperature monitoring, ${ }^{10}$ power reduction during energy delivery on the posterior wall, intracardiac echocardiography, ${ }^{11}$ esophageal cooling, ${ }^{12}$ and the administration of proton pump inhibitors after the procedure. ${ }^{13}$ These techniques differ in terms of their risks and benefits, and also in terms of practicality. In addition, none of these strategies is likely to be proven to be safer than the others given the extremely low prevalence of AE fistula. While real-time imaging of the esophagus with barium is practical, the results of this study suggest that it may also be accomplished without significantly increasing the risk of the ablation procedure.

\section{Prior Studies}

An earlier study showed that real-time esophagraphy is feasible in patients undergoing a catheter ablation procedure for $\mathrm{AF}^{3}$ However, there were only 50 patients in that study and no control group was available for comparison. This study shows that in large series of patients, barium administration does not appear to increase the risk of complications as compared to historical controls.

\section{Limitations}

One of the limitations of this retrospective study is that chest radiography was not performed in every patient following the ablation procedure. However, the diagnosis of aspiration pneumonitis is often based on clinical grounds since there is no gold standard. Objective findings on chest radiography may or may not be present, ${ }^{4}$ especially if the aspirate is small and/or the inflammatory response is mild. An important caveat of the study is that the lack of increased risk was likely related to the fact that sedation was withheld until administration of barium, and that only a small amount of barium paste was used.

In the surgical literature, the risk of pulmonary aspiration in a series of patients undergoing elective surgery was 1 in 3,886 patients $(0.03 \%)$. Therefore, another limitation is that the small sample size in this study was insufficient to detect a difference between the 2 groups given the extremely low prevalence of aspiration. Lastly, the results of this study do not apply to patients undergoing the ablation procedure under general anesthesia.

\section{Clinical Implications}

The main clinical implication of this study is that realtime imaging of the esophagus may be performed in patients undergoing a LA ablation procedure without increased risk. This strategy allows the operator to tailor the lesion set in each patient, thereby minimizing the risk of thermal injury to the esophagus. In patients who undergo barium-guided LA ablation of AF and the esophagus is thus avoided, fever within 24 hours of the procedure does not herald the presence of AE fistula.

\section{References}

1. Pappone C, Oral H, Santinelli V, Vicedomini G, Lang CC, Manguso F, Torracca L, Benussi S, Alfieri O, Hong R, Lau W, Hirata K, Shikuma N, Hall B, Morady F: Atrio-esophageal fistula as a complication of percutaneous transcatheter ablation of atrial fibrillation. Circulation 2004;109:2724-2726.

2. Scanavacca MI, D'Avila A, Parga J, Sosa E: Left atrial-esophageal fistula following radiofrequency catheter ablation of atrial fibrillation. J Cardiovasc Electrophysiol 2004;15:960-962.

3. Good E, Oral H, Lemola K, Han J, Tamirisa K, Igic P, Elmouchi D, Tschopp D, Reich S, Chugh A, Bogun F, Pelosi F Jr, Morady F: Movement of the esophagus during left atrial catheter ablation for atrial fibrillation. J Am Coll Cardiol 2005;46:2107-2110.

4. Warner MA, Warner ME, Weber JG: Clinical significance of pulmonary aspiration during the perioperative period. Anesthesiology 1993;78:5662 .

5. Oral H, Scharf C, Chugh A, Hall B, Cheung P, Good E, Veerareddy S, Pelosi F Jr, Morady F: Catheter ablation for paroxysmal atrial fibrillation: Segmental pulmonary vein ostial ablation versus left atrial ablation. Circulation 2003;108:2355-2360.

6. Oral H, Chugh A, Good E, Wimmer A, Dey S, Gadeela N, Sankaran S, Crawford T, Sarrazin JF, Kuhne M, Chalfoun N, Wells D, Frederick M, Fortino J, Benloucif-Moore S, Jongnarangsin K, Pelosi F Jr, Bogun F, Morady F: Radiofrequency catheter ablation of chronic atrial fibrillation guided by complex electrograms. Circulation 2007;115:2606-2612.

7. Kennedy R, Good E, Oral H, Huether E, Bogun F, Pelosi F, Morady F, Chugh A: Temporal stability of the location of the esophagus in patients undergoing a repeat left atrial ablation procedure for atrial fibrillation or flutter. J Cardiovasc Electrophysiol 2008;19:351-355. 
8. Dagres N, Kottkamp H, Piorkowski C, Doll N, Mohr F, Horlitz M, Kremastinos DT, Hindricks G: Rapid detection and successful treatment of esophageal perforation after radiofrequency ablation of atrial fibrillation: Lessons from five cases. J Cardiovasc Electrophysiol 2006; 17:1213-1215.

9. Hall B, Shah A, Huang D, Rosero S, Daubert J: Visualization of the esophagus during catheter ablation of atrial fibrillation. J Interv Card Electrophysiol 2005;13:135-137.

10. Cummings JE, Schweikert RA, Saliba WI, Burkhardt JD, Brachmann J, Gunther J, Schibgilla V, Verma A, Dery M, Drago JL, Kilicaslan $\mathrm{F}$, Natale A: Assessment of temperature, proximity, and course of the esophagus during radiofrequency ablation within the left atrium. Circulation 2005;112:459-464.

11. Ren JF, Lin D, Marchlinski FE, Callans DJ, Patel V: Esophageal imaging and strategies for avoiding injury during left atrial ablation for atrial fibrillation. Heart Rhythm 2006;3:1156-1161.

12. Tsuchiya $T$, Ashikaga $K$, Nakagawa $S$, Hayashida $K$, Kugimiya $H$ : Atrial fibrillation ablation with esophageal cooling with a cooled waterirrigated intraesophageal balloon: A pilot study. J Cardiovasc Electrophysiol 2007; 18:145-150.

13. Bunch TJ, Day JD: Novel ablative approach for atrial fibrillation to decrease risk of esophageal injury. Heart Rhythm 2008;5:624-627. 